\title{
Análise de Sensibilidade da Taxa de Acidente de uma Planta Industrial por Cadeias de Markov e Teoria de Perturbação Generalizada (GPT)
}

\author{
Eduardo F. de Lima ${ }^{1}$ \\ Danielle G. Teixeira ${ }^{2}$ \\ Paulo Fernando F. Frutuoso e Melo ${ }^{3}$ \\ Fernando C. da Silva ${ }^{4}$ \\ Programa de Engenharia Nuclear, COPPE, UFRJ, Rio de Janeiro, RJ
}

\begin{abstract}
Resumo.A GPT (Teoria da Perturbação Generalizada) é aplicada à análise de confiabilidade de um sistema com 3 canais de proteção iguais de uma planta industrial. Verifica-se a influência da taxa de demanda $(\nu)$ e da fração de falhas de causa comum sobre a frequência de acidente. A análise direta de sensibilidade já foi usada para estudar a influência destes parâmetros, em que se resolve o sistema de equações diferenciais oriundo da abordagem por Cadeias de Markov. Obtém-se a frequência de acidentes em função da taxa de demanda e da fração de falhas de causa comum. Com a GPT, a obtenção destas curvas torna-se mais simples. Para o intervalo $\nu<1000 /$ ano, cálculos de GPT com aproximações de $3^{\mathrm{a}}$ e $5 \underline{\mathrm{a}}$ ordens foram melhores que os com a aproximação de $1 \underline{\mathrm{a}}$ ordem. Para $\nu>1000 /$ ano a aproximação de $1^{\mathrm{a}}$ ordem teve um comportamento melhor que as de $3^{\underline{\mathrm{a}}}$ e $5^{\underline{\mathrm{a}}}$ ordens.
\end{abstract}

Palavras-chave. Canais de proteção, Frequência de acidentes, Cadeias de Markov, Confiabilidade, GPT.

\section{Introdução}

A GPT é um método heurístico usado na engenharia nuclear [2]. A aplicação de GPT em análise de confiabilidade, baseando-se em Cadeias de Markov, é discutida em [1]. A frequência de acidente de uma planta equipada com um canal de proteção em função da taxa de demanda do sistema é analisada em [3]. Diversos trabalhos, como [4], comprovam a importância de se determinar a frequência de acidente de um sistema em função da taxa de demanda. Estendendo a aplicação da GPT, este trabalho faz a análise de sensibilidade da frequência de acidente em uma planta com um sistema de três canais redundantes de proteção. Devido à redundância, é necessário levar em consideração a ocorrência de falhas de causa comum, para cujo tratamento existem modelos tais o dos Parâmetros Básicos,

\footnotetext{
${ }^{1}$ lima@nuclear.ufrj.br

${ }^{2}$ dteixeira@nuclear.ufrj.br

${ }^{3}$ frutuoso@nuclear.ufrj.br

${ }^{4}$ fernando@nuclear.ufrj.br
} 
das Letras Gregas Múltiplas e o do fator $\alpha$ [5]. Neste trabalho, foi usado este último, pela facilidade de obtenção de valores de seus parâmetros na prática.

\section{O Problema de 3 Canais}

O diagrama de transição com uma lógica de falha $2-3: F$ é mostrado na Fig.1. Esta lógica de falha significa que a falha do sistema ocorrerá se pelo menos 2 dos 3 canais falharem $(\mathrm{F}=$ falha $)$. O retângulo pontilhado contém os estados de falha que seriam considerados se o reparo fosse on-line, ou seja o reparo só é feito com a planta desligada. Assim, não são considerados os estados 6,9 e 10. Os parâmetros do terno $\langle i, j, l\rangle$ representam $i=$ número de canais em funcionamento, $j=$ número de canais com falha não revelada e $l=$ número de canais com falha revelada, quando o seu reparo é efetuado. $\lambda_{k}$ é a taxa de falha simultânea de $k$ canais idênticos, obtida do modelo $\alpha$ pelo uso do parâmetro $\alpha_{k}$ que representa a probabilidade de falha simultânea de $k$ dos $m$ canais idênticos, $\mu$ é a taxa de reparo de um canal, $\nu$ é a taxa de demanda do sistema, $\gamma$ é probabilidade de reparo de um canal não ser adequadamente feito e o número de equipes de reparo é igual ao de canais. As taxas $\lambda_{m}, \mu$ e $\nu$ são constantes, o que implica que os tempos de falha, de reparo e de demanda seguem distribuições exponenciais.

O modelo $\alpha$ [5] permite calcular $\lambda_{k}$ através da expressão $\lambda_{k}=\frac{k}{\left(\begin{array}{c}m-1 \\ k-1\end{array}\right)} \frac{\alpha_{k}}{\alpha_{T}} \lambda$ em que estamos considerando que o teste dos $\mathrm{m}=3$ canais é simultâneo. $\lambda$ é a taxa de falha total, que inclui falhas independentes e de causa comum, $\alpha_{T}=\sum_{k=1}^{m} k \alpha_{k}$ e $\sum_{k=1}^{m} \alpha_{k}=1$. A equação diferencial que governa o sistema é dada por:

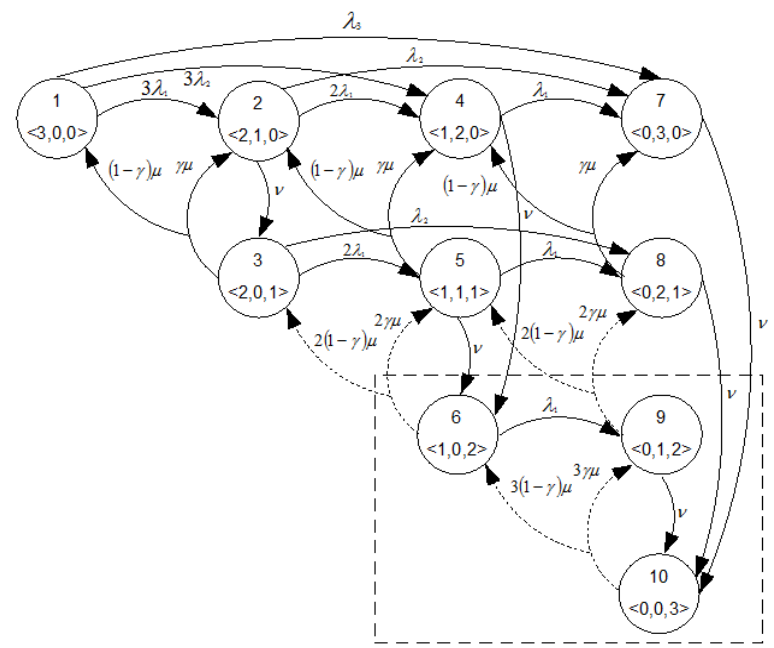

Figura 1: Diagrama de transição para 3 canais iguais usando uma lógica de falha $2-3: F$.

$$
\underline{\dot{p}}=M \underline{p}(t)
$$


onde $p(t) \equiv\left[\begin{array}{lll}p_{1}(t) & \ldots & p_{10}(t)\end{array}\right]^{T}$ e $p_{s}(t), s=1,2 \ldots, 10$, é a probabilidade do sistema estar no estado $i$ em $t$ e $M$ é a matriz de transição, construída considerando as transições de estado mostradas na Fig.1. Por exemplo, $M_{2,4}=2 \lambda_{1}$, que representa a taxa de transição do estado 2 para o 4 . O fator 2 nesta taxa de transição significa que, como há 2 canais funcionando no estado 2 e um estado 4 e ocorre somente uma falha, existem duas possibilidades desta falha ocorrer.

Admitindo que os canais estejam operacionais em $t=0$, teremos $\underline{p}(0)=\left[\begin{array}{llll}1 & 0 & \ldots & 0\end{array}\right]^{T}$.

Como a lógica de falhas é $2-3: F$, a frequência de acidente da planta $\eta$, será [4] (não estamos levando em conta o reparo on-line dos canais):

$$
\eta=\frac{\nu}{\tau_{p}} \int_{0}^{\tau_{p}}\left[p_{4}(t)+p_{5}(t)+p_{7}(t)+p_{8}(t)\right] d t .
$$

A equação (2) pode se escrita como:

$$
\eta=\int_{0}^{\tau_{p}} \underline{h}^{+^{T}} \underline{p}(t) d t
$$

onde $\underline{h}^{+^{T}}=\frac{n}{\tau_{p}} \underline{p}_{T}$ e $\underline{p}_{T}=\left[\begin{array}{llllllllll}0 & 0 & 0 & 1 & 1 & 0 & 1 & 1 & 0 & 0\end{array}\right]^{T}$.

A perturbação em $\alpha_{1}$ e $\nu$ dará uma frequência $\eta^{\prime}$ por uma expansão em série de Taylor:

$$
\begin{array}{r}
\eta^{\prime}=\eta+\sum_{n=1}^{\infty} \frac{1}{n !}\left\{\frac{\partial^{n} \eta}{\partial \alpha_{1}^{n}}\left(\delta \alpha_{1}\right)^{n}+\frac{\partial^{n} \eta}{\partial \nu^{n}}(\delta \nu)^{n}\right\}+ \\
\sum_{n=2}^{\infty} \frac{1}{n !}\left[\sum_{m=1}^{n-1}\left(\begin{array}{c}
n \\
m
\end{array}\right)\left\{\frac{\partial^{n} \eta}{\partial \alpha_{1}^{n-m} \partial \nu^{m}}\left(\delta \alpha_{1}\right)^{n-m}(\delta \nu)^{m}\right\}\right]
\end{array}
$$

onde, a partir da equação (3), tem-se:

$$
\begin{aligned}
& \frac{\partial^{n} \eta}{\partial \alpha_{1}^{n}}=\int_{0}^{\tau_{p}} \underline{h}^{+^{T}} \frac{\partial^{n} \underline{p}(t)}{\partial \alpha_{1}^{n}} d t . \\
& \frac{\partial^{n} \eta}{\partial \nu^{n}}=\int_{0}^{\tau_{p}} \underline{h}^{+^{T}} \frac{\partial^{n} \underline{p}(t)}{\partial \nu^{n}} d t+ \begin{cases}\frac{n}{\nu} \eta ; & \text { se } n=1 \\
\frac{\eta}{\nu} \int_{0}^{\tau_{p}} \underline{h}^{+^{T}} \frac{\partial^{n-1} \underline{p}(t)}{\partial \nu^{n-1}} d t ; & \text { se } \quad n>1\end{cases} \\
& \frac{\partial^{n} \eta}{\partial \alpha_{i}^{n-m} \partial \nu^{m}}=\int_{0}^{\tau_{p}} \underline{h}^{+^{T}} \frac{\partial^{n} \underline{p}(t)}{\partial \alpha_{1}^{n-m} \partial \nu^{m}} d t+ \begin{cases}\frac{1}{\nu} \int_{0}^{\tau_{p}} \underline{h}^{+^{T}} \frac{\partial^{n-1} \underline{p}(t)}{\partial \alpha_{1}^{n-1}} d t ; & \text { se } m=1 \\
\frac{m}{\nu} \int_{0}^{\tau_{p}} \underline{h}^{+^{T}} \frac{\partial^{n-1} \underline{p}(t)}{\partial \alpha_{1}^{n-m} \partial \nu^{m-1}} d t ; & \text { se } m>1\end{cases}
\end{aligned}
$$

As derivadas de $\underline{p}(t)$ em relação aos parâmetros $\alpha_{1}$ e $\nu$ são soluções das equações:

$$
\frac{d}{d t}\left(\frac{\partial^{n}}{\partial \alpha_{1}^{n}} \underline{p}(t)\right)=M\left(\frac{\partial^{n}}{\partial \alpha_{1}^{n}} \underline{p}(t)\right)+\underline{S}_{1}^{(n)}(t)
$$




$$
\begin{gathered}
\frac{d}{d t}\left(\frac{\partial^{n}}{\partial \nu^{n}} \underline{p}(t)\right)=M\left(\frac{\partial^{n}}{\partial \nu^{n}} \underline{p}(t)\right)+\underline{S}_{\nu}^{(n)}(t) \\
\frac{d}{d t}\left(\frac{\partial^{n}}{\partial \alpha_{1}^{n-m} \partial \nu^{m}} \underline{p}(t)\right)=M\left(\frac{\partial^{n}}{\partial \alpha_{1}^{n-m} \partial \nu^{m}} \underline{p}(t)\right)+\underline{S}_{1 \nu}^{(n-m, n)}(t)
\end{gathered}
$$

cujos termos fonte são dados por:

$$
\begin{gathered}
\underline{S}_{1}^{(n)}(t)=M_{1}\left\{a_{n} \underline{p}(t)+\sum_{i=1}^{n-1}\left(\begin{array}{c}
n \\
i
\end{array}\right) a_{i}\left(\frac{\partial^{n-i}}{\partial \alpha_{1}^{n-1}} \underline{p}(t)\right)\right\} \\
\underline{S}_{\nu}^{(n)}(t)=M_{\nu}\left\{\underline{p}(t)+\sum_{i=1}^{n-1}\left(\begin{array}{c}
n \\
i
\end{array}\right) \frac{\partial^{n-i}}{\partial \nu^{n-i}} \underline{p}(t)\right\} \\
\underline{S}_{1 \nu}^{(n-m, m)}(t)=M_{1}\left\{\sum_{i=1}^{n-1}\left(\begin{array}{c}
n-m \\
l
\end{array}\right) a_{i}\left(\frac{\partial^{n-l}}{\partial \alpha_{1}^{n-m-l} \partial \nu^{m}} \underline{p}(t)\right)+a_{i} \frac{\partial^{m}}{\partial \nu^{m}} \underline{p}(t)\right\}+ \\
m M_{\nu}\left(\frac{\partial^{n-l}}{\partial \alpha_{1}^{n-m} \partial \nu^{m-1}} \underline{p}(t)\right)
\end{gathered}
$$

onde: $a_{i}=\frac{i ! 2^{i-1}}{\left(3-\left[2 \alpha_{1}+\alpha_{2}\right]\right)^{i}}, \operatorname{com} i=1,2,3 \mathrm{e}$

$$
M_{1}=2 M^{\prime}+\lambda M_{\lambda}^{(1)} .
$$

A matriz $M_{\nu}$ é obtida da matriz $M$ derivando os elementos desta última em relação à taxa de demanda $\nu$. Os elementos da matriz $M_{\lambda}^{1}$ são obtidos a partir dos da matriz $M$ derivando os desta última em relação à taxa de falha $\lambda_{1}$. A matriz $M^{\prime}$ é obtida da matriz $M$ preservando os elementos desta última que envolvem taxas de falha $\lambda_{m}$ e tornando nulos todos os demais.

Pela Relação de Reciprocidade de Fontes [6], as equações (5)-(7) se tornam:

$$
\begin{gathered}
\int_{0}^{\tau_{p}} \underline{h}^{+^{T}} \frac{\partial^{n} \underline{p}(t)}{\partial \alpha_{1}^{n}} d t=\int_{0}^{\tau_{p}} \underline{p}^{*^{T}}(t) \underline{S}_{1}^{n}(t) d t \\
\int_{0}^{\tau_{p}} \underline{h}^{+^{T}} \frac{\partial^{n} \underline{p}(t)}{\partial \nu^{n}} d t=\int_{0}^{\tau_{p}} \underline{p}^{*^{T}}(t) \underline{S}_{\nu}^{n}(t) d t \\
\int_{0}^{\tau_{p}} \underline{h}^{+^{T}} \frac{\partial^{n} \underline{p}(t)}{\partial \alpha_{1}^{n-m} \nu^{m}} d t=\int_{0}^{\tau_{p}} \underline{p}^{*^{T}}(t) \underline{S}_{1 \nu}^{n-m, n}(t) d t
\end{gathered}
$$

onde $\underline{p}^{*}(t)$, a função importância associada à quantidade integral $\eta$, é solução da equação:

$$
-\frac{d}{d t} \underline{p}^{*}(t)=M^{T} \underline{p}^{* T}(t) \underline{h}^{+T}
$$


Substituindo as equações (15), (16) e (17) nas equações (5), (6) e (7), respectivamente, e as equações resultantes na equação (4) obtém-se:

$$
\begin{aligned}
& \eta^{\prime}=\eta+\sum_{n=1}^{\infty} \frac{1}{n !}\left\{\left(\int_{0}^{\tau_{p}} \underline{p}^{*^{T}}(t) \underline{S}_{1}^{(n)}(t) d t\right)\left(\delta \alpha_{1}\right)^{n}+\left(\int_{0}^{\tau_{p}}{\underline{p^{*}}}^{*^{T}}(t) \underline{S}_{\nu}^{(n)}(t) d t\right)(\delta \nu)^{n}\right\}+ \\
& \sum_{n=2}^{\infty} \frac{1}{n !}\left[\left(\begin{array}{c}
n \\
m
\end{array}\right)\left\{\left(\int_{0}^{\tau_{p}} \underline{p}^{*^{T}}(t) \underline{S}_{1 \nu}^{(n-m, m)}(t) d t\right)\left(\delta \alpha_{1}\right)^{n-m}(\delta \nu)^{m}\right\}\right]+ \\
& \frac{1}{\nu} \eta(\delta \nu)+ \\
& \frac{1}{\nu} \sum_{n=2}^{\infty} \frac{n}{n !}\left\{\left(\int_{0}^{\tau_{p}} \underline{p}^{*^{T}}(t) \underline{S}_{\nu}^{(n-1)}(t) d t\right)(\delta \nu)^{n}+\left(\int_{0}^{\tau_{p}} \underline{p}^{*^{T}}(t) \underline{S}_{1}^{(n-1)}(t) d t\right)\left(\delta \alpha_{1}\right)^{n-1}(\delta \nu)\right\}+ \\
& \frac{1}{\nu} \sum_{n=3}^{\infty} \frac{1}{n !}\left\{\sum_{m=2}^{n-1}\left(\begin{array}{c}
n \\
m
\end{array}\right) m\left(\int_{0}^{\tau_{p}} \underline{p}^{*^{T}}(t) \underline{S}_{1 \nu}^{(n-m, m)}(t) d t\right)\left(\delta \alpha_{1}\right)^{n-m}(\delta \nu)^{m}\right\}
\end{aligned}
$$

onde $\underline{S}_{1}, \underline{S}_{\nu}$ e $\underline{S}_{1 \nu}$ foram definidas nas equações (11), (12) e (13).

\section{Resultados}

O uso da GPT na análise de sensibilidade da frequência de acidente $\eta$ de uma planta (usando um sistema de 3 canais de proteção idênticos), foi feito perturbando os parâmetros $\nu$ e $\alpha_{1}$. Usamos como tempo de missão $\left(\tau_{p}\right) 1$ ano; taxa de falha $(\lambda)$ de 10 /ano; taxa de reparo $(\mu)$ de $365 /$ ano e a probabilidade de falha no reparo $(\gamma)$ de $1 \%$, conforme [7]. Além disso, os parâmetros $\alpha_{1}$ e $\alpha_{2}$ foram assim tratados: $\alpha_{1}=0,7+0,05(n-1)$; para $n=1, \ldots, 5$ e $\alpha_{2}=0,1$, considerando-se 0,8 como o valor de referência para $\alpha_{1}$. Para cada valor de $\alpha_{1}$, foram adotadas variações em $\nu$ de acordo com a Tabela 1 . Como a faixa de variação da taxa de demanda é muito grande, a Tabela 1 apresenta os 12 casos que foram abordados para a análise do problema. Cada caso representa um intervalo de variação, para o qual foi definido um valor de referência, empregado para o cálculo direto da taxa de acidente, usando a equação (3), à qual nos referimos nos gráficos dos resultados (que comentaremos a seguir) como solução direta. Os cálculos de sensibilidade usando GPT significam resolver o sistema de equações diferenciais da equação (1) 60 vezes, pois foram adotados 5 valores de referência do parâmetro $\alpha_{1}$ e, para cada valor de referência, o sistema foi resolvido para 12 taxas de demanda de referência (ver Tabela 1). Comparativamente, os resultados apresentados em [8] necessitaram de solução do mesmo sistema mais de 600 vezes.

As aproximações de $3^{\underline{a}}$ e $5^{\underline{a}}$ ordens foram melhores para $\nu<1000 /$ ano, enquanto que para $\nu>1000 /$ ano, a aproximação de $1 \underline{a}$ ordem foi melhor (ver a Figura 2(a) e 2(b), que representam os resultados para $\alpha_{1}=0.75$ e $\alpha_{2}=0.9$ ). Para $\nu<1000 /$ ano, a frequência de acidente em função da taxa de demanda cresce rapidamente [8], havendo a necessidade de se usar altas ordens da série de Taylor para $\eta$ neste intervalo. Diante disso, as aproximações 
de $3^{\mathrm{a}}$ e $5^{\mathrm{a}}$ ordens foram melhores enquanto a aproximação de $1^{\mathrm{a}}$ ordem chegou a ter um desvio de até $8,8 \%$ para $\nu=100 /$ ano.

Tabela 1: Variações da taxa de demanda $\nu$.

\begin{tabular}{|c|c|c|c|}
\hline Caso & Intervalo, $\nu(/$ ano $)$ & $\nu$ Referência & Parâmetro $\nu$ perturbado \\
\hline 1 & $1 \leq \nu \leq 9$ & 5 & $1+1(n-1) ; n=1, \ldots, 9$ \\
\hline 2 & $10 \leq \nu \leq 50$ & 30 & $10+5(n-1) ; n=1, \ldots, 9$ \\
\hline 3 & $55 \leq \nu \leq 95$ & 75 & $55+5(n-1) ; n=1, \ldots, 9$ \\
\hline 4 & $100 \leq \nu \leq 300$ & 200 & $100+25(n-1) ; n=1, \ldots, 9$ \\
\hline 5 & $300 \leq \nu \leq 500$ & 400 & $300+25(n-1) ; n=1, \ldots, 9$ \\
\hline 6 & $500 \leq \nu \leq 750$ & 625 & $500+25(n-1) ; n=1, \ldots, 11$ \\
\hline 7 & $750 \leq \nu \leq 1000$ & 875 & $750+25(n-1) ; n=1, \ldots, 11$ \\
\hline 8 & $1000 \leq \nu \leq 2000$ & 1500 & $1000+250(n-1) ; n=1, \ldots, 5$ \\
\hline 9 & $2000 \leq \nu \leq 3000$ & 2500 & $2000+250(n-1) ; n=1, \ldots, 5$ \\
\hline 10 & $3000 \leq \nu \leq 4000$ & 3500 & $3000+250(n-1) ; n=1, \ldots, 5$ \\
\hline 11 & $4000 \leq \nu \leq 6000$ & 5000 & $4000+250(n-1) ; n=1, \ldots, 9$ \\
\hline 12 & $6000 \leq \nu \leq 10000$ & 8000 & $6000+500(n-1) ; n=1, \ldots, 9$ \\
\hline
\end{tabular}

Para $\nu>1000 /$ ano, a função é praticamente assintótica [8] e suas derivadas de ordens superiores são próximas de zero. Como a aproximação de 1a ordem não usa essas derivadas, seus resultados são melhores neste intervalo. As aproximações de $3 \underline{a}$ e $5 \underline{a}$ ordens usam-nas e, portanto, tendem a aumentar o seu desvio, chegando a $12,6 \%$ para $\nu=6500$ /ano na aproximação de $5^{\mathrm{a}}$ ordem. Existe também a dificuldade de se trabalhar com números tão pequenos (devido à aproximação da curva a uma assíntota), aumentando o erro dos resultado.
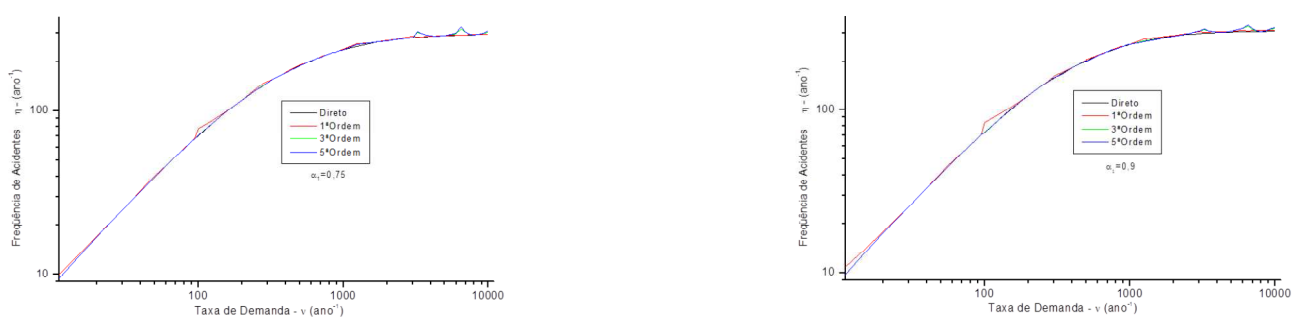

(a) Frequência de acidentes em função de taxa de de- (b) Frequência de acidentes em função de taxa de demanda de $\alpha_{1}=0.75$ manda de $\alpha_{1}=0.9$

Figura 2: Frequência de acidentes em função de taxa de demanda (a) e (b) 


\section{Conclusões}

Este trabalho analisou a sensibilidade de um sistema de proteção de três canais considerando como quantidade integral a taxa de acidente da planta. Esta redundância, torna os cálculos extensivos quando feitos através da abordagem tradicional. A análise da sensibilidade do sistema foi bastante satisfatória, sendo recomendada a GPT para tal abordagem. Pode-se verificar que as aproximações de $1^{\mathrm{a}}$, $3^{\mathrm{a}}$ e $5^{\underline{\mathrm{a}}}$ ordens tiveram bons resultados em relação ao cálculo direto.

\section{Referências}

[1] A. Gandini, Importance and sensitivity analysis in assessing system reliability, IEEE Transactions on Reliability. V 39, pp. 61-70, 1990.

[2] F. C. Silva, and A. Gandini, Perturbation techniques for reactor life cycle analysis, Proceedings of the International Topical Meeting on Advances in Reactor Physics, Mathematics and Computation, Paris, France, pp. 1253-1258, 1987.

[3] P. F. Frutuoso e Melo, A . C. M. Alvim and F. C. Silva, Sensitivity Analysis on the Accident Rate of a Plant Equipped with a Single Protective Channel by Generalized Perturbation Methods, Annals Nuclear Energy V.25, pp.1191-1207, 1998.

[4] P. F. Frutuoso e Melo, L. F. Oliveira, and R.Youngblood, Markovian models for the reliability analysis of multichannel protective systems.Proceedings of II International conference on Probabilistic Safety Assessment and Management, San Diego, CA, 1994.

[5] A. Mosleh, and N. O. Siu, A multi-parameter common cause failure model, In: Proceedings of the 9th International Conference on Structural Mechanics in Reactor Technology, Vol. M1, pp. 147-152, Lausanne, Switzerland, 1987.

[6] F. C. Silva, Desenvolvimento de métodos da teoria de perturbação generalizada (GPT) e suas aplicações à física de reatores. Tese de Doutorado, COPPE/UFRJ, Rio de Janeiro, 1989.

[7] Oliveira, L. F., Youngblood, R. and Frutuoso e Melo, P. F., Hazard rate of a plant equipped with a two-channel protective system subject to a high demand rate, Reliability Engineering and System Safety, V. 28, pp.35-58, 1990.

[8] P. F. Frutuoso e Melo, L. F. Oliveira, R.Youngblood, A Markovian model for the reliability analysis of multichanneled protective systems considering revealed failures and common-cause failures by the alpha model. In: Anais do IX Encontro Nacional de Física de Reatores e Termohidráulica. Rio de Janeiro, RJ: Associação Brasileira de Energia Nuclear, p.440-446, 1993. 